\title{
Control of artificial strain energy in Abaqus
}

\author{
Xiaoming Chen ${ }^{1, a}$, Jin Duan ${ }^{1}$, Yungui Li ${ }^{1}$, Jianyun Sun ${ }^{1}$ \\ ${ }^{1}$ China state construction technical center, Beijing, China \\ aHanee@126.com
}

\begin{abstract}
Keywords: Artificial strain energy, time-history, elastic-plastic, Abaqus
Abstract. Reduced integral is used widely to improve the efficiency of solver especially for nonlinear analysis at the cost of additional artificial strain energy, therefore, proper approach should be used to control the hourglass effect induced by reduced integral. Numerical results show that for Abaqus/Standard solver, stiffness mode of hourglass control can exhibit much better performance than the enhanced mode, but the enhanced method was the only proposal of Abaqus while using analysis continuation techniques. Results also show that the Standard solver may not present reasonable results under low level damping, thus the damping mode should be chosen carefully for structural analysis.
\end{abstract}

\section{Introduction}

Reduced integral method is widely used in finite element analysis. This method can improve the computing efficiency significantly especially for nonlinear analysis, on the other hand, the elements used reduced integral can present much more accuracy.

Both the accuracy and the insensitivity to mesh distortion all depend on the complementary order of assumed displacement polynomials of elements. The integral order of precise numerical integral is determined by those terms of the highest order of elemental polynomials, but few of those non-complementary terms of element can exhibit positive roles, therefore, reduced integral method was researched by Zienkiewicz [1] and Hughes [2,3] in 1970s to filter the effect of these terms to improve the property of elements.

On the other hand, for those displacement-based elements formulated by using the principle of minimum potential energy, their solutions may exhibit lower-bound theorem [4] because of the overestimated structural stiffness, proper reduced integral can also modify this phenomenon.

But reduced integral would destroy the elemental complementary, and then the rank of the stiffness matrix would be decreased, this may lead to redundant zero energy modes which is also called hourglass effect. To control the hourglass effect, additional stabilization matrix should be introduced to control this artificial strain energy.

Both complete integral and reduced integral elements are available in FEA software Abaqus, such as the general shell elements S4 and S4R [5]. In the nonlinear analysis under seismic waves for building structures, reduced integral method has to be used to shorten the time consuming, therefore, hourglass control method must be chosen properly to ensure the energy conservation of the whole system, otherwise, it would induced failure solutions.

Numerical method was used to research the hourglass effect for shear wall structures in this paper. Numerical results show that although Abaqus proposed various forms of control approach, the artificial strain energy induced by hourglass effect may still be significant, it can even lead to failure results for those systems with low damping level. Different influence factors including mesh size and different damping modes are also researched to reveal the proper hourglass control in building structure analysis.

\section{Control approaches for artificial strain energy in Abaqus}

Reduced integral will produce loss of stiffness for the elements, and a specific deformation may be induced without resisting stresses, this situation is called hourglass modes. Several methods are available in Abaqus for suppressing hourglass modes, these methods for hourglass control attempts to 
minimize these problems without introducing excessive constraints on the element's physical response, as described below:

(1) Integral viscoelastic approach for Explicit

The integral viscoelastic approach available in Abaqus/Explicit generates more resistance to hourglass forces early in the analysis step where sudden dynamic loading is more probable.

(2) Kelvin viscoelastic approach for Explicit

Three methods are defined as Kelvin viscoelastic approach for Explicit solver, including pure stiffness, pure viscous approach and combination of stiffness and viscous. The pure stiffness form is available for all reduced-integration elements and is recommended for both quasi-static and transient dynamic simulations, both the pure viscous form and the linear combination form are available only for solid and membrane elements with reduced integration.

(3) Total stiffness approach for Standard

The total stiffness method is the default hourglass control approach for all first-order, reduced-integration elements, but it is only available in Standard.

(4) Enhanced hourglass control approach for both Standard and Explicit

The enhanced hourglass control is the only available approach for both Standard and Explicit which represents a refinement of the pure stiffness method based on the enhanced assumed strain method. As the enhanced hourglass control approach is compatible between Abaqus/Standard and Abaqus/Explicit, It is recommended that enhanced hourglass control to be used for both Abaqus/Standard and Abaqus/Explicit for all import analyses. Then it is the preferable option for structural analysis under seismic waves.

\section{Model of shear wall}

As shown in Fig.1, free vibration of the shear wall was analyzed, for which the horizontal force was removed suddenly from its top. The time-histories of top displacement are researched for different damping models. The time-histories of both total internal energy and artificial strain energy are also presented for the reliability of results. C40 concrete is used. Four kinds of damping modes are used as follows:

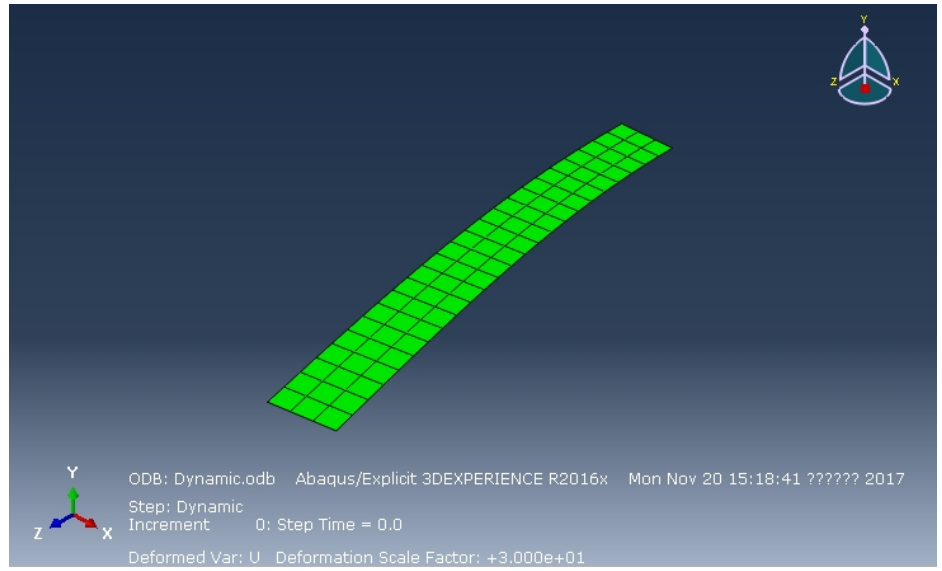

Fig.1 Model of shear wall with $1 \mathrm{~m}$ meshes

(a) Both Rayleigh mass proportional damping and stiffness proportional damping; (b) Only Rayleigh mass proportional damping; (c) Only stiffness proportional damping; (d) Free vibration without damping.

The mass proportional ratio and stiffness proportional ration of Rayleigh damping are derived by using both the primary period and the second order period with the damping ratio of 0.05 [6].

\section{Results of initial model}

The initial model was meshed into $1 \mathrm{~m}$ with S4R, that means reduced integration method was used. The Young's modulus is $32.5 \mathrm{MPa}$ [7]. For reduced integral model, enhanced hourglass control approach is used. Time-histories of internal energy and artificial energy are shown in Fig.2 to Fig.5. 


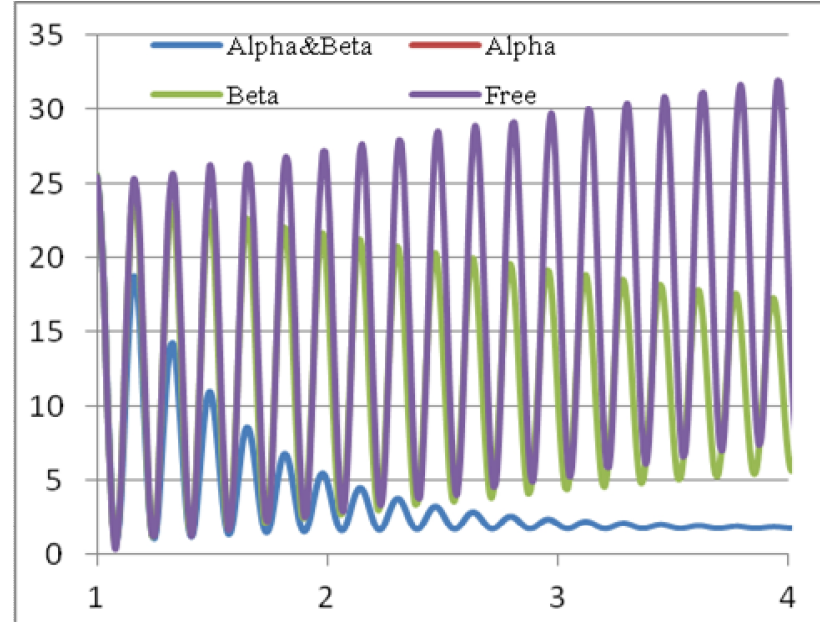

Fig.2 Internal energy of Standard(Initial)

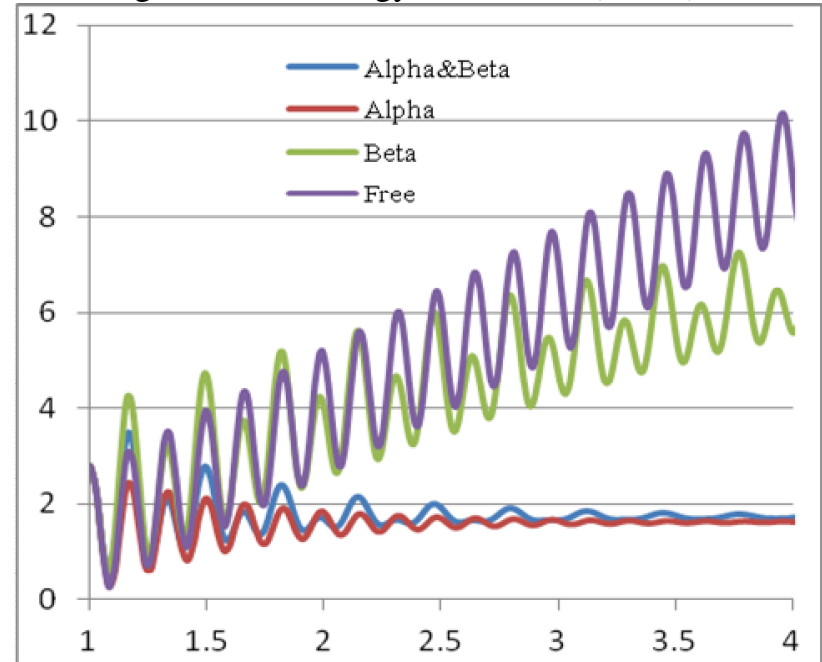

Fig.4 Artificial energy of Standard(Initial)

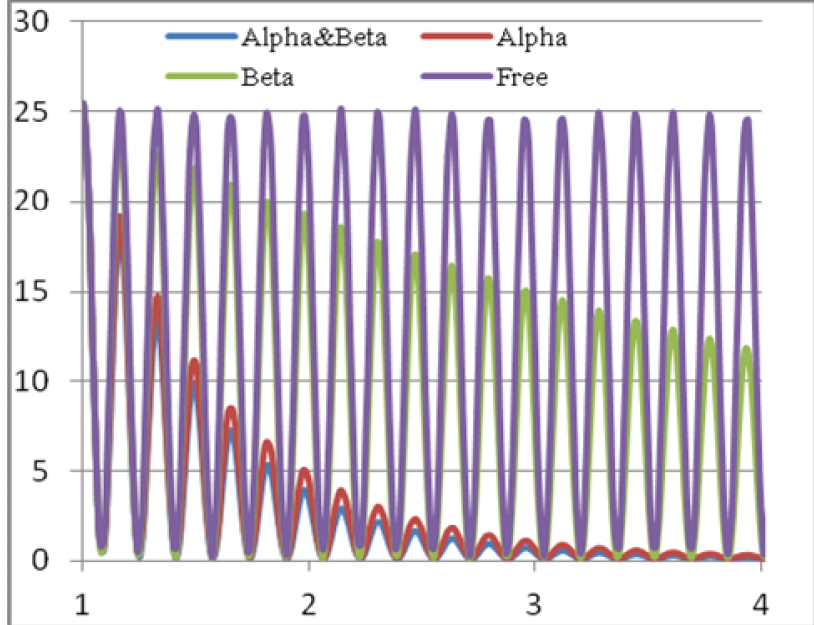

Fig.3 Internal energy of Explicit(Initial)

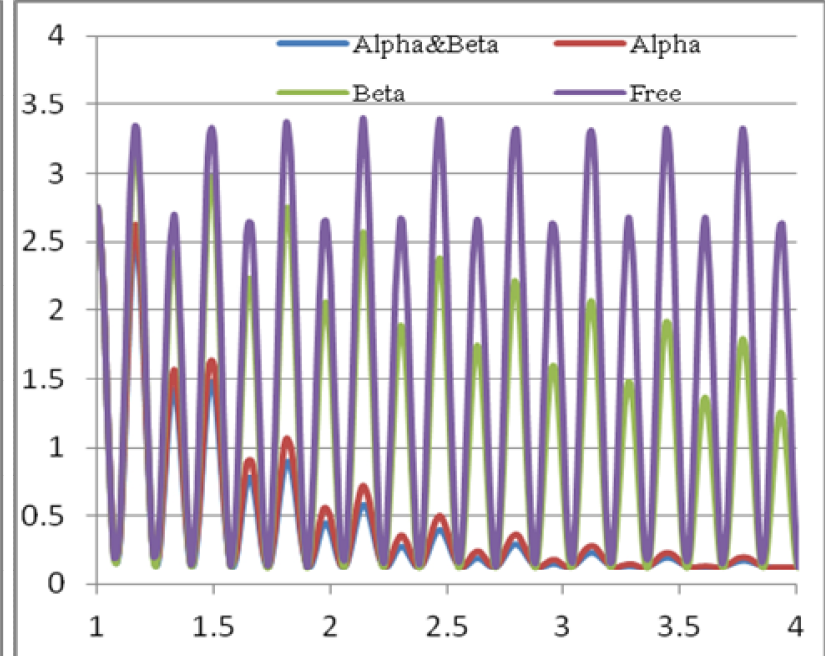

Fig.5 Artificial energy of Explicit(Initial)

Results of the initial model show that because of the artificial energy induced by hourglass effect, Abaqus/Standard solver cannot present reasonable solutions under low damping level. The enhanced hourglass control can exhibit satisfied performance in Abaqus/Explicit solver.

\section{Effect of mesh size}

The shear wall of initial model shown in Fig. 1 was remeshed into $0.5 \mathrm{~m}$ to research artificial energy induced by hourglass effect, which are shown in Fig.6 to Fig.9.

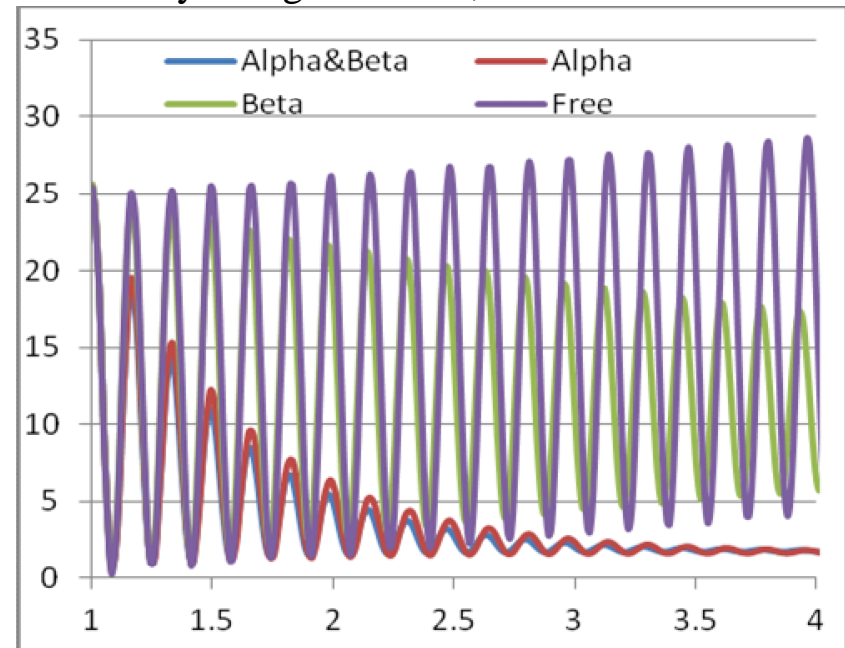

Fig.6 Internal energy of Standard(Mesh $0.5 \mathrm{~m}$ )

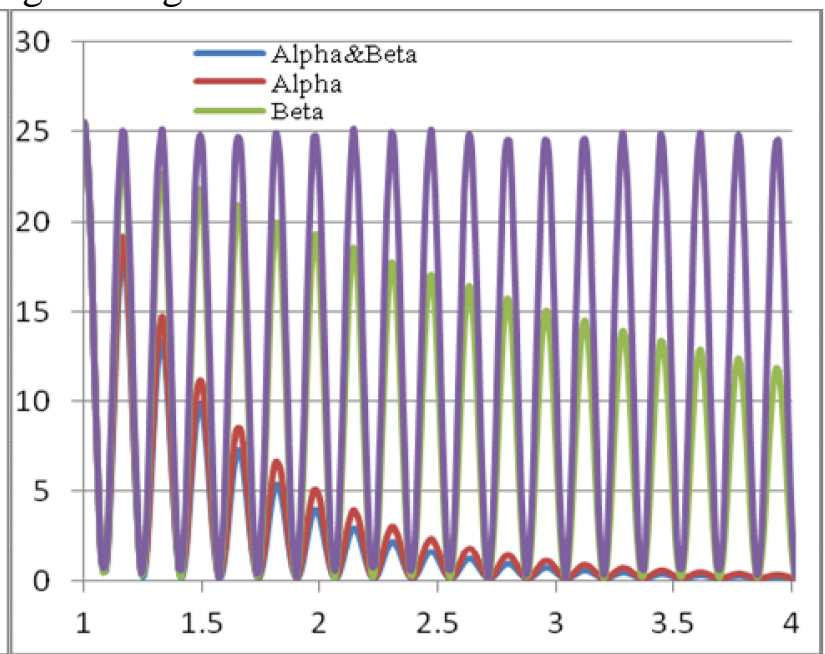

Fig.7 Internal energy of Standard(Mesh $0.5 \mathrm{~m}$ ) 

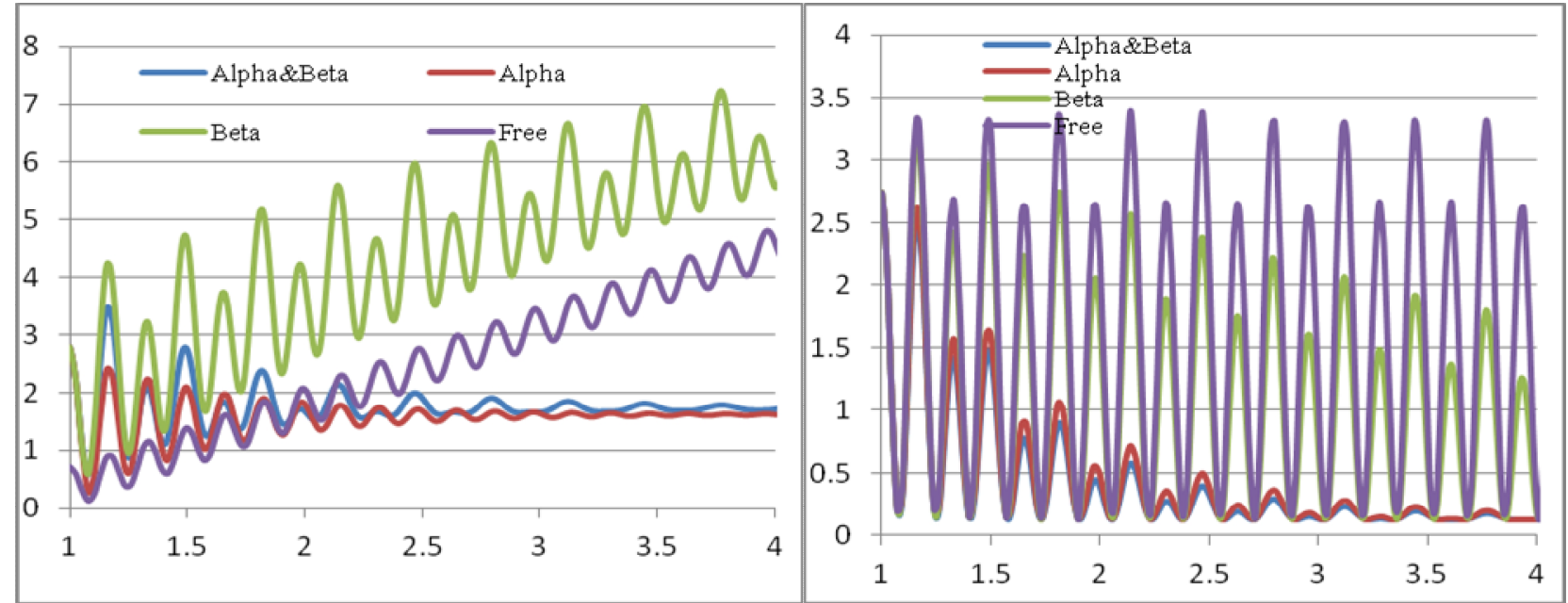

Fig.8 Artificial energy of Standard(mesh $0.5 \mathrm{~m}$ )

Fig.9 Artificial energy of Explicit(mesh $0.5 \mathrm{~m}$ )

As proposed by some research, hourglass effect can be improved by using refined grids. These results mean that the artificial energy has been reduced more or less for the Standard solver, and for the Explicit solver, results of energy are still stable for each damping model.

\section{Complete integral method}

As reduced integral was used widely for increasing the efficiency of solver especially for nonlinear analysis as the cost of hourglass effect, then complete integral was used as alternative to present structural internal energy and artificial energy (Fig.10 to Fig.13).
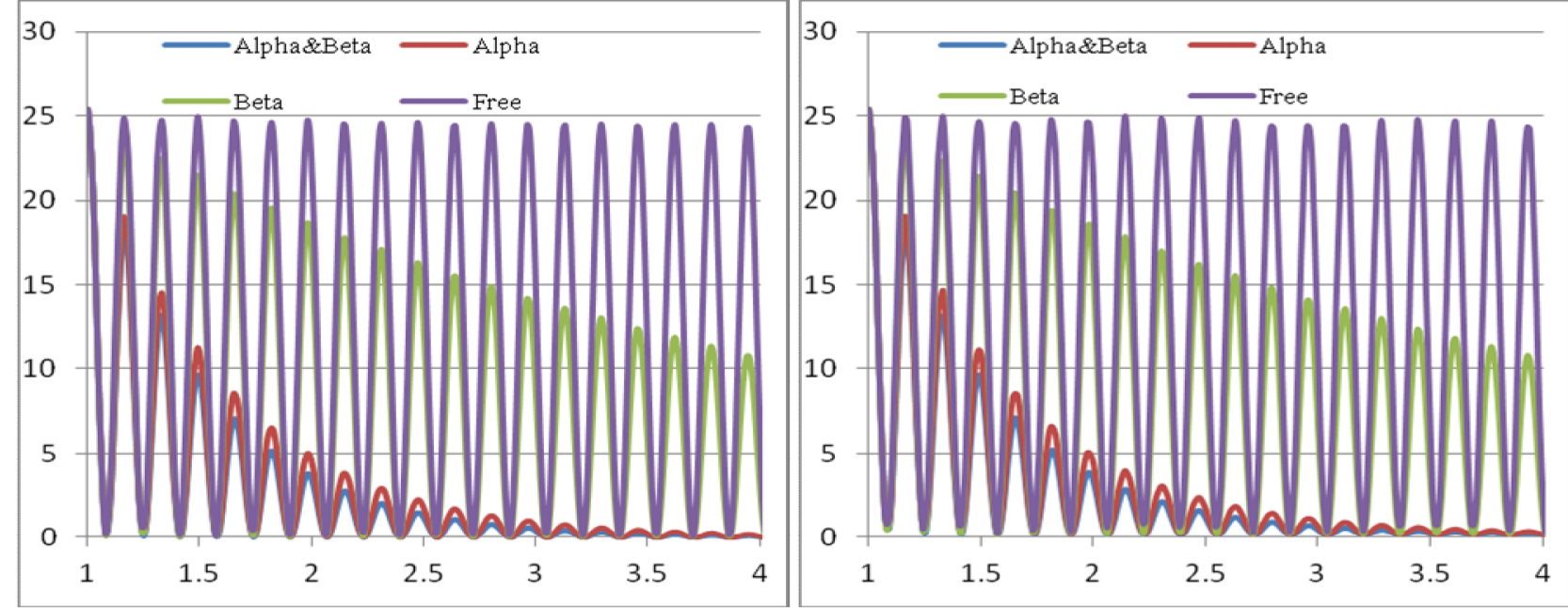

Fig.10 Internal energy of Standard(Complete integral) Fig.11 internal energy of Explicit(Complete integral)
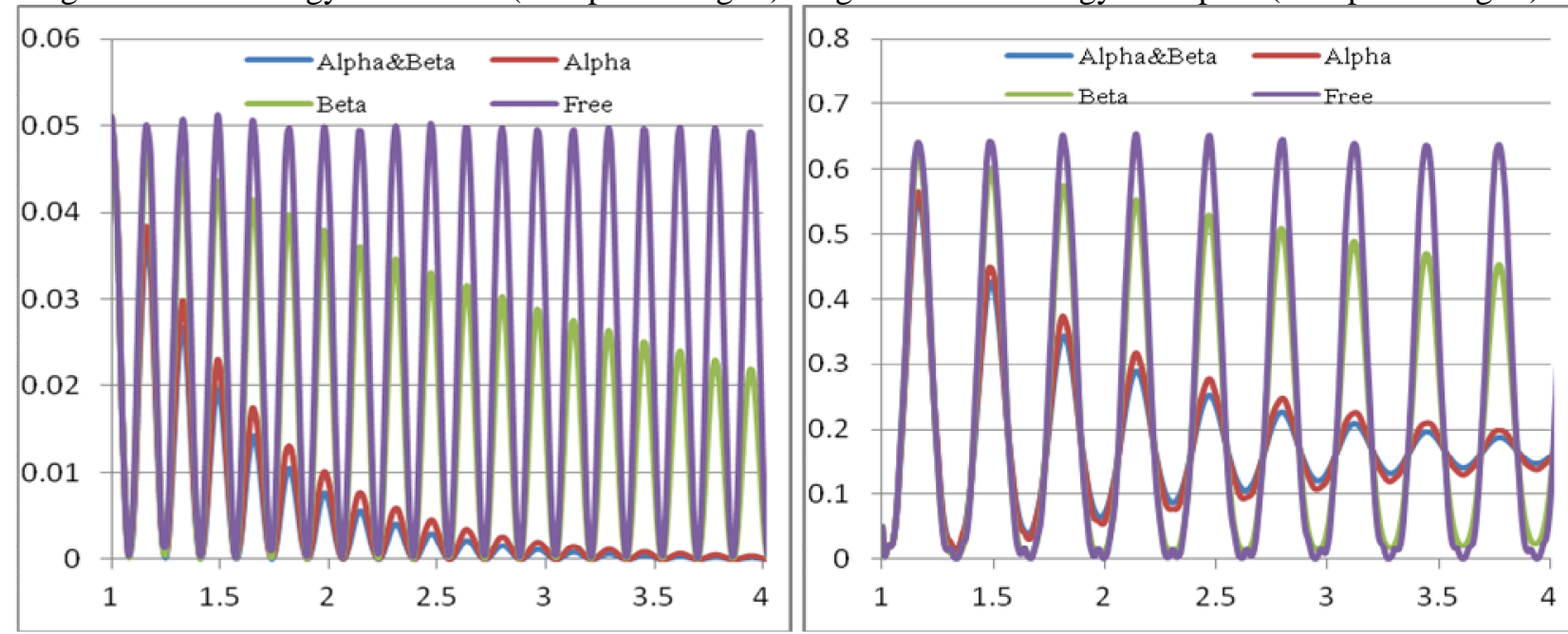

Fig.12 Artificial energy of Standard(Complete integral) Fig.13 Artificial energy of Explicit(Complete integral) 
It can be seen that the artificial energy of both Standard and Explicit solver can be neglected by using complete integral method.

\section{Stiffness mode for hourglass control}

Results presented above show that enhanced hourglass control cannot exhibit ideal solutions, thus the stiffness mode was tried together with reduced integral method to analyze the same model. Time-histories of internal energy are presented in Fig.14 and Fig.15.

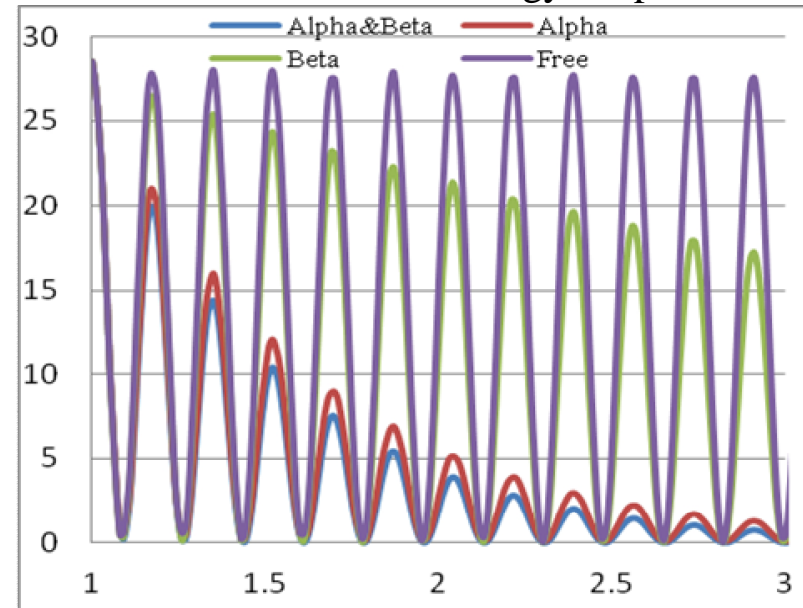

Fig.14 Internal energy of Standard(Stiffness mode)

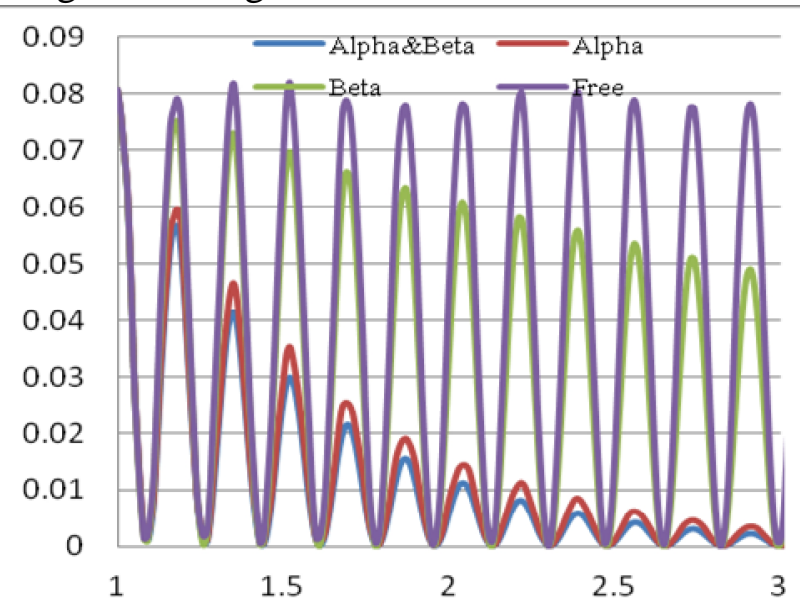

Fig.15 Artificial energy of Standard(Stiffness mode)

Compared with enhanced method, the stiffness method can exhibit perfect effect on hourglass control for Standard, but it's a pity that this method cannot be used for Explicit.

\section{Conclusions}

Reduced integral is used widely to improve the efficiency of solver especially for nonlinear analysis, at the same time, proper approach should be introduced to control the hourglass effect induced by reduced integral which would lead to more or less artificial strain energy. Numerical results show that the stiffness mode of hourglass control can exhibit much better performance than the enhanced mode in Abaqus/Standard solver. The enhanced method proposed by Abaqus for analysis continuation techniques, the standard solver may not present reasonable results under low level damping, thus the damping mode should be chosen carefully.

\section{References}

[1] Zienkiewicz, O. C. Taylor, R. L.; Too, J. M.: Reduced integration technique in general analysis of plates and shells. Int. J. Num. Meth. Eng. 3(1971), 275-290.

[2] Hughes, T. J. R.; Cohen, M. Haroun, M.: Reduced and selective integration techniques in the finite element analysis of plate. Nucl. Eng. Des. 46(1978), 203-222

[3] Hughes, T. J. R. Mixed finite element methods - reduced and selective integration techniques: A unification of concepts. Comp. Meth. Appl. Mech. Eng. 15(1978), 63-81

[4] Wang Xucheng. Finite element method. Tsinghua university press, Beijing, China, 2003.

[5] ABAQUS Inc. ABAQUS User Manual, V2016(2016).

[6] EL. Wilson. Three-Dimensional Static and Dynamic Analysis of Structures: a Physical Approach with Emphasis on Earthquake Engineering. Computers and Structures (1998).

[7] China architecture \& building press. Code for design of concrete structures. Beijing, China (2010). 